\title{
Effective Date Range
}

National Cancer Institute

\section{Source}

National Cancer Institute. Effective Date Range. NCI Thesaurus. Code C93560.

The date and time span for when the entity is active. 